\title{
Correction to "Analysis and Optimization of Sleeping Mode in WiMAX via Stochastic Decomposition Techniques"
}

\author{
Amar Prakash Azad, Member, IEEE, Sara Alouf, and Eitan Altman, Fellow, IEEE
}

This note is to correct the authorlist and authors affiliations of [1]. The submitted version of this paper that appeared in the September 2011 issue of the IEEE Journal on Selected Areas in Communications was missing two co-authors: Sara Alouf and Eitan Altman. The byline and author affiliations should have appeared as in the current correction.

Also, the following information about funding was missing from [1]:

\section{ACKNOWLEDGEMENTS}

This work was partly supported by the ANR WINEM and by the Indo-French Centre for the Promotion of Advanced Research (IFCPAR), project 4000-IT.

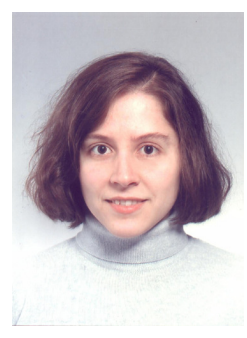

Sara Alouf received an M.Sc. in computer networking and distributed systems in 1999 and a $\mathrm{Ph} . \mathrm{D}$. in computer science in 2002, both from the University of Nice Sophia Antipolis. During 20032004, she was a post-doctoral fellow at the Free University at Amsterdam, within the Optimization of Business Processes research group. Since March 2004, she has been with INRIA working as a full time researcher in the MAESTRO project-team. She is currently involved in the common lab between INRIA and Alcatel-Lucent Bell Labs. She has served in the technical program committees of ACM Sigmetrics, ACM MobiHoc, IEEE Infocom, Performance, ITC, Valuetools, IEEE Globecom and WIOPT. She received the best paper award of the IFIP Wireless Days 2009 conference. Her research interests include modeling and performance evaluation of communication networks. More information can be found at http://www-sop.inria.fr/members/Sara.Alouf/.

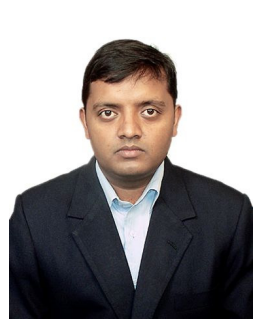

Amar Prakash Azad received MS in Electrical Communication Engineering from Indian Institute of Science, India, in 2006, and PhD at INRIA, France, in 2010. Currently, he is a researcher at University of California Santa Cruz, USA. He has been visiting researcher at University of Illinois Urbana Champaign (USA), and University of California Berkeley (USA), Supelec (Paris), Tata Institute of Fundamental Research (Bombay, INDIA). His research interests are optimal resource management, game theory and its application, and security in wireless networks. His work received best paper award in IFIP Wireless days in 2009 and was in top 15 papers at IEEE WoWMoM 2007. He is also a recipient of MHRD fellowship from 2003 to 2006. He served as TPC for several conferences including Globecom '07, '08, '09, WCNC '10, Sigmetrics '10 (Shadow), Infocom '10 (Workshop). He was also a peer reviewer for several journals including TON, JSAC, Comnet, Elsevier etc. More information can be found at http://sites.google.com/site/amarazad2/.

At the time of submission of [1], all three authors were affiliated with INRIA, Sophia Antipolis, France. A. P. Azad is currently with UCSC, USA Email: amar.azad@ieee.org, \{Sara.Alouf,Eitan.Altman\}@inria.fr.

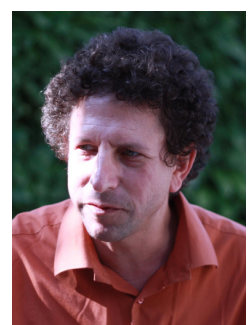

Eitan Altman received the B.Sc. degree in electrical engineering (1984), the B.A. degree in physics (1984) and the Ph.D. degree in electrical engineering (1990), all from the Technion-Israel Institute, Haifa. In (1990) he further received his B.Mus. degree in music composition in Tel-Aviv university. Since 1990, Dr. Altman has been a researcher at INRIA (National research institute in computer science and control) in Sophia-Antipolis, France. He has been in the editorial boards of several scientific journals: Wireless Networks (WINET), Computer Networks (COMNET), Computer Communications (Comcom), J. Discrete Event Dynamic Systems (JDEDS), SIAM J. of Control and Optimisation (SICON), Stochastic Models, and Journal of Economy Dynamic and Control (JEDC). He received the best paper award in the Networking 2006, in Globecom 2007 and in IFIP Wireless Days 2009 conferences, and is a coauthor of two papers that have received the best student paper awards (at QoFis 2000 and at Networking 2002). His areas of interest include networking, stochastic control and game theory. More information can be found at http://www-sop.inria.fr/members/Eitan.Altman/.

\section{REFERENCES}

[1] A. P. Azad, "Analysis and optimization of sleeping mode in WiMAX via stochastic decomposition techniques," IEEE Journal on Selected Areas in Communications, vol. 29, no. 8, pp. 1630-1640, September 2011, special issue on Energy-Efficient Wireless Communications. http://dx.doi.org/10. 1109/JSAC.2011.110912. 